phys. stat. sol. (a) 145, 509 (1994)

Subject classification: 73.60 and $74.60 ;$ S5.11

Institute of Physics, Slovak Academy of Sciences, Bratislava $\left.{ }^{1}\right)$ (a), Physikalisches Institut der Universität Karlsruhe $\left.{ }^{2}\right)(b)$, and

Laboratoire de Physique du Solide, URA au CNRS, DO 155, Université Nancy I, Vandoeuvre-les-Nancy ${ }^{3}$ ) (c)

\title{
Superconductivity and Critical Fields in Amorphous Tungsten/Silicon Multilayers
}

\author{
By \\ E. Majkova (a), S. Luby (a), M. Jergel (a), H. v. Löhneysen (b), \\ C. Strunk (b), and B. George (c)
}

The superconducting properties of amorphous $\mathrm{W} / \mathrm{Si}$ multilayers are studied. A nearly linear increase of $T_{\mathrm{c}}$ with increasing number of W/Si bilayers $N$ is observed for $N=3$ to 10 . The parallel and perpendicular critical field data show strong interlayer coupling between the superconducting layers in a $(1 \mathrm{~nm} \mathrm{Si} / 1 \mathrm{~nm} \mathrm{~W}) \times 10$ multilayer and weak coupling for a $(5 \mathrm{~nm} \mathrm{Si} / 1 \mathrm{~nm} \mathrm{~W}) \times 9$ multilayer. The broadening of the resistive transition in perpendicular magnetic fields is analyzed within the frame of the thermally activated flux flow model. A temperature independent activation energy $U$ is found for weakly coupled $\mathrm{W} / \mathrm{Si}$ multilayers with a magnetic field dependence $U \sim-\ln H$.

\section{Introduction}

Artificially prepared superconducting layered systems of superconductor/insulator (S/I) type offer a good opportunity to study the effect of coupling between superconducting layers on the superconducting properties. The coupling strength can be changed effectively by varying the insulating-layer thickness. 2D superconductivity and dimensional transitions in the critical fields induced by variation of the interlayer coupling strength were investigated with various multilayer $S / I$ systems [1 to 3]. Interest in the behaviour of $2 \mathrm{D}$ coupled superconductors has increased considerably since the high- $T_{c}$ superconductors were discovered. Recently, the observed increase of $T_{c}$ with increasing number of bilayers in $\mathrm{YBaCuO} / \mathrm{Pr} \mathrm{BaCuO}$ superlattices was explained by interlayer coupling $[4,5]$. The vortex properties in high- $T_{\mathrm{c}}$ superconductors and superlattices with different interlayer couplings are extensively studied at present [ 5 to 7]. The experimental data hint at a close correlation between yortex behaviour and interlayer coupling strength. The effect of interlayer coupling on vortex motion in $\mathrm{MoGe} / \mathrm{Ge}$ multilayer was reported by White et al. [8]. An effect similar to that observed in high- $T_{\mathrm{c}}$ superconductors was found. The authors observed a crossover from a vortex motion affected by the interlayer coupling to a regime where the vortex segments moved independently. Therefore, the study of vortex motion in superconducting multilayers with different strengths of interlayer coupling is of considerable interest.

In our previous study [9] superconductivity with superconducting transition temperature $T_{\mathrm{c}}$ up to $4.2 \mathrm{~K}$ was found in amorphous $\mathrm{W} / \mathrm{Si}$ multilayers (W/Si MLs). $T_{\mathrm{c}}$ of bulk tungsten

1) Dubravska cesta 9,84228 Bratislava, Slovakia.

2) D-76128 Karlsruhe, Federal Republic of Germany.

$\left.{ }^{3}\right)$ B.P. 239, F-54506 Vandoeuvre-les-Nancy, France. 
is only $0.012 \mathrm{~K}[10]$. We ascribed the superconductivity to an amorphous W-Si phase which is formed at W/Si interfaces during the deposition and/or subsequent heat treatment. (The formation and growth of amorphous silicide phases was discussed by Maex [11].) As was shown in [12], the presence of pure amorphous tungsten in our W/Si MLs with tungsten layers 1 to $2 \mathrm{~nm}$ thick does not seem very probable. Experiments on electronic transport revealed very different behaviour of the sheet resistance of our W/Si MLs in comparison with pure amorphous tungsten films prepared under the same deposition conditions. A weak temperature dependence of the sheet resistance with $R_{\mathrm{sq}}(290) / R_{\mathrm{sq}}(30)$ ratio between 0.85 and 0.97 and the onset of superconducting fluctuations around $10 \mathrm{~K}$ were found for the W/Si MLs. The ratio $R_{\mathrm{sq}}(290) / R_{\mathrm{sq}}(30)=1.009$ and no tendency towards superconducting transition was observed for a pure W film. Here, at low temperatures $(T<30 \mathrm{~K})$ the dependence of $R_{\mathrm{sq}}$ on $T$ typical for $2 \mathrm{D}$ disordered metallic films with logarithmic increase of $\Delta R_{\mathrm{sq}} / R_{\mathrm{sq}}^{2}$ with decreasing temperature was found. Hence, we assume that our W/Si MLs are simple layered systems of superconductor/insulator type with superconducting amorphous W-Si layers and intermediate insulating Si layers.

The aim of this work is to study the superconductivity, the effect of interlayer coupling on $T_{c}$, the upper critical fields, and vortex motion in W/Si MLs with different insulating-layer thickness.

\section{Experimental Procedures}

W/Si MLs were electron beam deposited in a vacuum of $10^{-7} \mathrm{~Pa}$. The oxidized $\mathrm{Si}(100)$ wafers with $\mathrm{SiO}_{2}$ layer $0.5 \mu \mathrm{m}$ thick were used as substrates held at $50^{\circ} \mathrm{C}$. The details of the deposition procedure are described elsewhere [9]. The tungsten layer thickness $d_{\mathrm{w}}$ ranged between 1 and $2 \mathrm{~nm}$, the silicon layer thickness $d_{S \mathrm{i}}$ between 1 and $15 \mathrm{~nm}$. Multilayers with the number of bilayers $N=3,6,9$, and 10 were prepared.

The presence of multilayered structures was verified by cross-sectional transmission electron microscopy (CS-TEM) and small-angle X-ray diffraction (SAXD) using a Stoe high resolution diffractometer. The structure of $W$ and $S i$ sublayers was examined using TEM and large-angle $\mathrm{X}$-ray diffraction (LAXD). $\mathrm{CuK}_{\alpha}$ radiation was used in the $\mathrm{X}$-ray experiments.

The sheet resistance was measured using a four-point probe in a liquid helium cryostat in the temperature range 1.2 to $300 \mathrm{~K}$. The perpendicular and parallel critical fields $H_{\mathrm{c} 2 \perp}$, $H_{\mathrm{c} 2 \|}$ were measured with magnetic fields perpendicular and parallel to the multilayered structure up to $5 \mathrm{~T}$ using a superconducting solenoid. The critical field was defined by $50 \%$ of the resistive transition.

\section{Results and Discussion}

The CS-TEM and SAXD experiments confirmed the multilayered structure of our samples (Fig. 1). From the SAXD pattern the actual value of the multilayer period $A=d_{\mathrm{W}}+d_{\mathrm{Si}}$ was calculated using the relation

$$
m \lambda=2 A\left(1-\frac{\delta}{\sin ^{2} \theta_{m}}\right) \sin \theta_{m},
$$

where $m$ is the satellite order, $\theta$ the Bragg angle, $1-\delta$ the real part of the mean index of refraction of the material, and $\lambda$ the $\mathrm{X}$-ray wavelength. The tungsten and silicon layer 


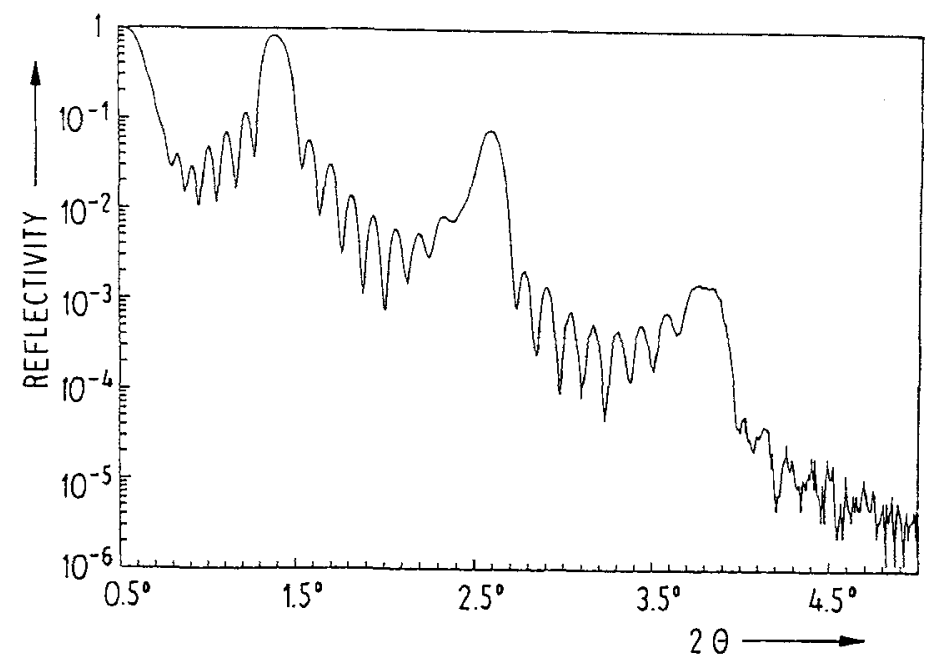

Fig. 1. Small-angle diffraction pattern of a $(5 \mathrm{~nm} \mathrm{Si} / 1 \mathrm{~nm} \mathrm{~W}) \times 9$ multilayer

thicknesses $d_{\mathrm{w}}$ and $d_{\mathrm{Si}}$ were derived from computer simulations of SAXD patterns [13] and the calculated values are given in Table 1. Both TEM and LAXD confirmed the amorphous structure of $\mathrm{W}$ and Si sublayers for all samples. As we have shown previously [9, 14], the evaporated tungsten is amorphous for $d_{\mathrm{w}} \leqq 4 \mathrm{~nm}$ at our deposition conditions. Silicon was found amorphous for all samples, i.e. for $d_{\mathrm{Si}} \leqq 15 \mathrm{~nm}$.

All multilayers were superconducting with $T_{\mathrm{c}}$ between 1.63 and $4.21 \mathrm{~K}$. The values of $T_{\mathfrak{c}}$, sheet resistance $R_{\mathrm{sq}}(290)$, and the $R_{\mathrm{sq}}(290) / R_{\mathrm{sq}}(30)$ ratio are given in Table 1 . $T_{\mathrm{c}}$ was found to increase with increasing number of bilayers from $1.63 \mathrm{~K}$ for $(5 \mathrm{~nm} \mathrm{Si} / 1 \mathrm{~nm} \mathrm{~W}) \times 3 \mathrm{ML}$ up to $2.73 \mathrm{~K}$ for $(5 \mathrm{~nm} \mathrm{Si} / 1 \mathrm{~nm} \mathrm{~W}) \times 10$ (Fig. 2). A similar dependence of $T_{\mathrm{c}}$ was observed for $\mathrm{Al}-\mathrm{Al}_{2} \mathrm{O}_{3}$ and $\mathrm{Sn}-\mathrm{SiO}_{x}$ layered structures [15], $\mathrm{NbSe}_{2}$ layered crystals [16], and, as mentioned above, for $\mathrm{YBaCuO} / \mathrm{PrBaCuO}$ superlattices $[4,5]$. This "size" effect hints at a coupling between the superconducting layers. The fact that the highest transition temperature $T_{\mathrm{c}}=4.21 \mathrm{~K}$ was obtained for the thinnest insulator barrier thickness $d_{\mathrm{Si}}=1 \mathrm{~nm}$ also supports our assumption. A depression of $T_{\mathrm{c}}$ with increasing insulating layer thickness was reported

Table 1

Parameters of $\mathrm{W} / \mathrm{Si}$ multilayers: modulation length $A, \mathrm{~W}$ and Si layer thicknesses $d_{\mathrm{W}}$ and $d_{\mathrm{Si}}$, sheet resistance at room temperature $R_{\mathrm{sq}}(290)$, superconducting transition temperature $T_{\mathrm{c}}$, and resistance ratio $R_{\mathrm{sq}}(290) / R_{\mathrm{sq}}(30)$

\begin{tabular}{lcccccc}
\hline $\begin{array}{l}\text { No. of sample } \\
\text { (nominal composition) }\end{array}$ & $\begin{array}{l}A \\
(\mathrm{~nm})\end{array}$ & $\begin{array}{l}d_{\mathrm{W}} \\
(\mathrm{nm})\end{array}$ & $\begin{array}{l}d_{\mathrm{si}} \\
(\mathrm{nm})\end{array}$ & \multicolumn{1}{l}{$\begin{array}{l}R_{\mathrm{sq}}(290) \\
(\Omega)\end{array}$} & $\begin{array}{l}T_{\mathrm{c}} \\
(\mathrm{K})\end{array}$ & $R_{\mathrm{sq}}(290) / R_{\mathrm{sq}}(30)$ \\
\hline $1(1 \mathrm{~nm} \mathrm{Si} / 1 \mathrm{~nm} \mathrm{~W}) \times 10$ & 1.9 & 0.9 & 1.0 & 107.8 & 4.21 & 0.972 \\
$2(5 \mathrm{~nm} \mathrm{Si} / 1 \mathrm{~nm} \mathrm{~W}) \times 3$ & 6.2 & 1.1 & 5.1 & 711.2 & 1.63 & 0.856 \\
$3(5 \mathrm{~nm} \mathrm{Si} / 1 \mathrm{~nm} \mathrm{~W}) \times 6$ & 6.3 & 1.1 & 5.2 & 248.7 & 2.17 & 0.903 \\
$4(5 \mathrm{~nm} \mathrm{Si} / 1 \mathrm{~nm} \mathrm{~W}) \times 9$ & 6.5 & 1.2 & 5.3 & 113.2 & 2.63 & 0.934 \\
$5(5 \mathrm{~nm} \mathrm{Si} / 1 \mathrm{~nm} \mathrm{~W}) \times 10$ & 6.2 & 1.1 & 5.1 & 152.2 & 2.73 & 0.907 \\
$6(8 \mathrm{~nm} \mathrm{Si} / 2 \mathrm{~nm} \mathrm{~W}) \times 10$ & 10.9 & 2.0 & 8.9 & 74.7 & 3.14 & 0.952 \\
$7(15 \mathrm{~nm} \mathrm{Si} / 2 \mathrm{~nm} \mathrm{~W}) \times 10$ & 14.9 & 2.3 & 12.6 & 102.1 & 2.84 & 0.962 \\
\hline
\end{tabular}




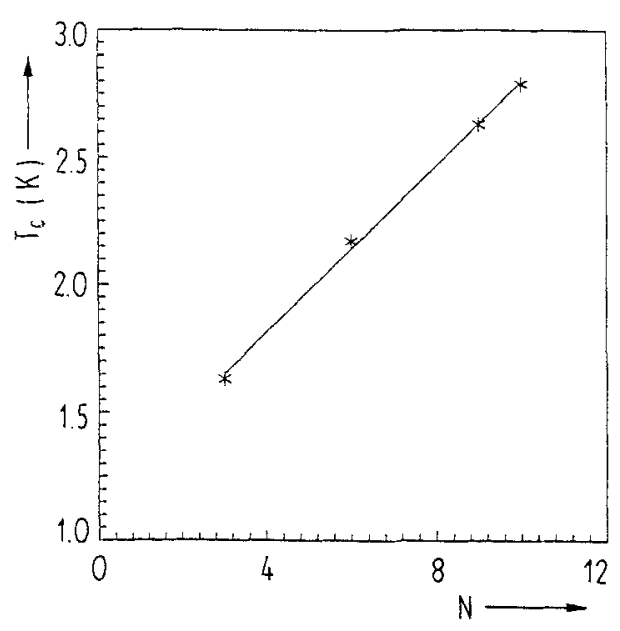

Fig. 2. Increase of the superconducting transition temperature $T_{\mathbf{c}}$ with increasing number of bilayers $N$ for the $(5 \mathrm{~nm} \mathrm{Si} / 1 \mathrm{~nm} \mathrm{~W}) \times N$ set

also for $\mathrm{YBaCuO} / \mathrm{PrBaCuO}$ superlattices $[4,17]$ and for $\mathrm{BiSrCaCuO} / \mathrm{BiSrCuO}$ MLs [18]. Recently, Michielsen et al. [19] reported that the size effect could be explained by involving a particular type of interlayer pairing interaction. According to their model, $T_{\mathrm{c}}$ of a $\mathrm{S} / \mathrm{I}$ multilayer stack of $N$ superconducting layers is

$$
T_{\mathrm{c}}(N) \approx T_{\mathrm{c}}(1) \cos \left(\frac{\pi}{N+1}\right) .
$$

Our results show rather a linear increase of $T_{\mathrm{c}}$ with increasing number of bilayers and no tendency to saturation was found up to ten bilayers. Assuming that pure amorphous tungsten is present in our MLs and the superconducting amorphous W-Si phase is formed at the $\mathrm{W} / \mathrm{Si}$ interfaces only, i.e. that the structure of our MLs is of $(\ldots \mathrm{Si} / \mathrm{a}-\mathrm{W}-\mathrm{Si} / \mathrm{a}-\mathrm{W} / \mathrm{a}-\mathrm{W}-\mathrm{Si} / \mathrm{Si}$. .) type, with 19 superconducting layers, this lack of saturation is even more surprising.

The value of $T_{\mathrm{c}}$ is also affected by the thickness of the intermixed region at the W/Si interfaces. The effect is well documented by the subsequent increase of $T_{\mathrm{c}}$ from 4.21 up to $4.6 \mathrm{~K}$ observed after annealing the $(1 \mathrm{~nm} \mathrm{Si} / 1 \mathrm{~nm} \mathrm{~W}) \times 10 \mathrm{ML}$ up to $650^{\circ} \mathrm{C}$ for $40 \mathrm{~s}$. Above this temperature the tungsten layers crystallize completely, the crystalline b.c.c. $\mathrm{W}$ and/or tetragonal $\mathrm{WSi}_{2}$ phases are formed and superconductivity disappears. During the heat treatment the mixed interlayers grow [14] and the thickness of the Si insulating layers decreases. Consequently, an increase of $T_{c}$ should be expected. This behaviour was indeed observed in our previous viork [20]. It was shown that when the thickness of the intermixed region formed at $\mathrm{W} / \mathrm{Si}$ interfaces during the deposition was reduced by oblique evaporation, a decrease of $T_{\mathrm{c}}$ from 2.84 to $1.76 \mathrm{~K}$ for MLs with similar nominal values of $d_{\mathrm{w}}$ and $d_{\mathrm{si}}$ was found.

The proximity effect often affects the value of $T_{\mathrm{c}}$ in layered systems of superconductor/metal type. The sheet resistance of Si layers in our W/Si MLs is $>10^{8} \Omega$, i.e. several orders of magnitude larger than that of conducting layers. Consequently the proximity coherence length can be assumed to be very small and the proximity effect is negligible [21].

The resistive transitions in parallel and perpendicular magnetic fields for samples $(1 \mathrm{~nm} \mathrm{Si} / 1 \mathrm{~nm} \mathrm{~W}) \times 10$ and $(5 \mathrm{~nm} \mathrm{Si} / 1 \mathrm{~nm} \mathrm{~W}) \times 9$ are shown in Fig. $3 \mathrm{a}, \mathrm{b}$ and $4 \mathrm{a}, \mathrm{b}$, respectively. The dependences of $H_{\mathrm{c} 21}, H_{\mathrm{c} 2 \|}$ on temperature of both samples are shown 


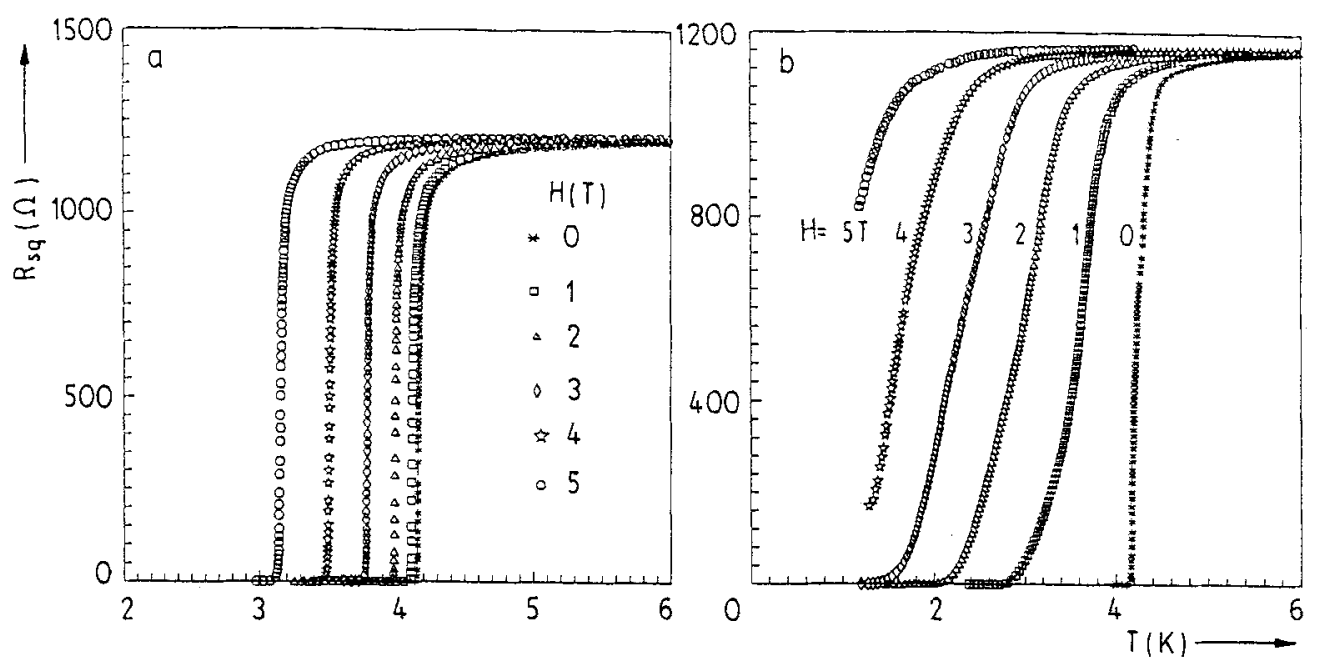

Fig. 3. Magnetic field dependence of the resistivity for magnetic field a) parallel and b) perpendicular to the layers for the $(1 \mathrm{~nm} \mathrm{Si} / 1 \mathrm{~nm} \mathrm{~W}) \times 10$ multilayer

in Fig. 5. Here, an increased anisotropy in critical fields with increasing Si layer thickness is found. This behaviour is typical for 2D layered superconductors [1]. Nearly linear temperature dependences of $H_{\mathrm{c} 2 \perp}(T)$ near $T_{\mathrm{c}}$ with the slopes $\beta=-1.59 \mathrm{~T} / \mathrm{K}$ for $(1 \mathrm{~nm} \mathrm{Si} /$ $1 \mathrm{~nm} \mathrm{~W}) \times 10$ and $\beta=-1.52 \mathrm{~T} / \mathrm{K}$ for $(5 \mathrm{~nm} \mathrm{Si} / 1 \mathrm{~nm} \mathrm{~W}) \times 9 \mathrm{MLs}$ are found. The slight positive curvature of $H_{\mathrm{c} 2 \perp}$ observed for both samples was reported for many layered superconductors and is supposed to originate from layering and/or localization and interaction effects [1].
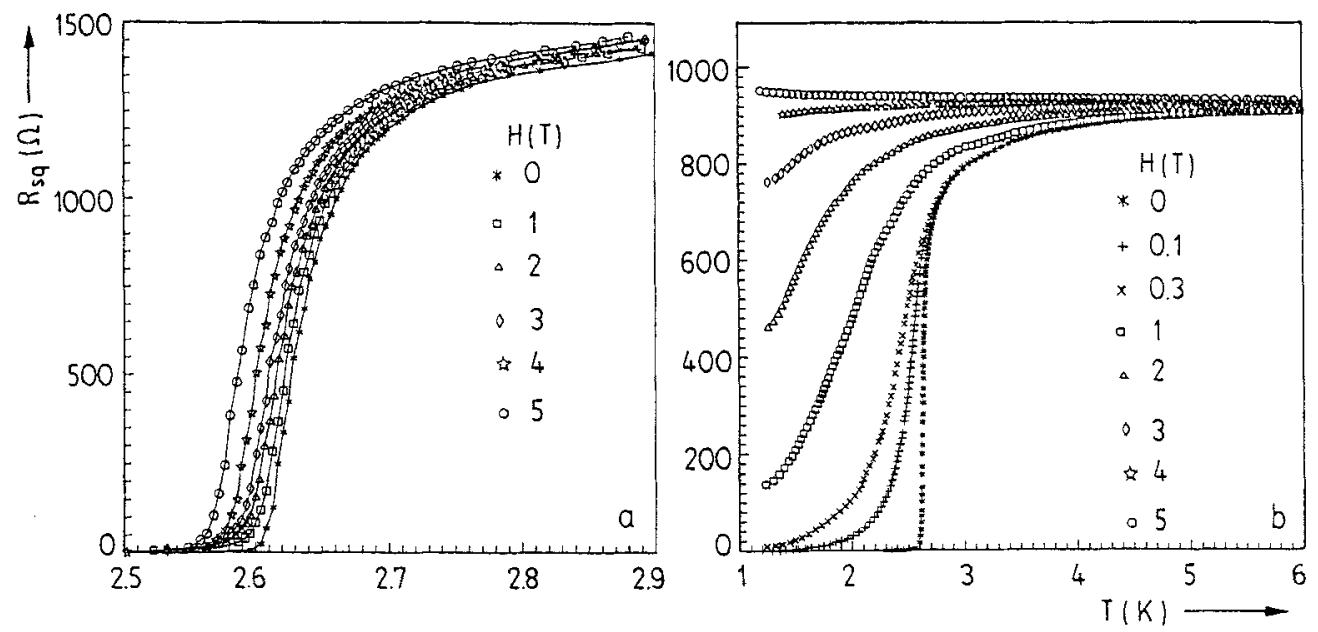

Fig. 4. Magnetic field dependence of the resistivity for magnetic field a) parallel and b) perpendicular to the layers for the $(5 \mathrm{~nm} \mathrm{Si} / 1 \mathrm{~nm} \mathrm{~W}) \times 9$ multilayer 


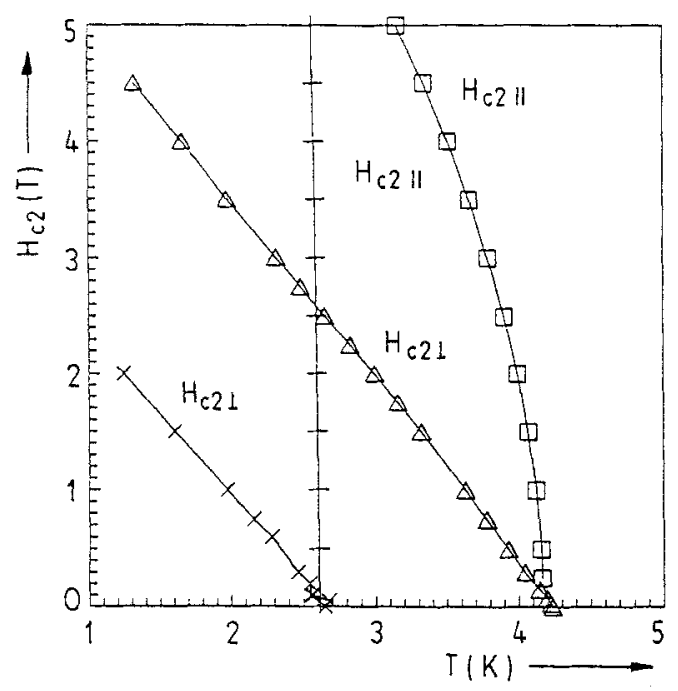

Fig. 5. Upper critical fields $H_{\mathrm{c} 2 \perp}, H_{\mathrm{c} 2 \text { if }}$ vs. $T$ for the $(1 \mathrm{~nm} \mathrm{Si} / 1 \mathrm{~nm} \mathrm{~W}) \times 10^{-}(\triangle, \square)$ and $(5 \mathrm{~nm} \mathrm{Si} / 1 \mathrm{~nm} \mathrm{~W}) \times 9(\times,+)$ multilayers

According to the Ginzburg-Landau theory [1] the coherence length $\xi_{\|}(T=0)$ can be calculated for dirty superconductors using the relation

$$
\xi_{\| !}^{2}(T=0)=\frac{\Phi_{0}}{2 \pi}\left\{\frac{1}{\frac{\mathrm{d} H_{\mathrm{c} 2 \perp}}{\mathrm{d} T} T_{\mathrm{c}}}\right\},
$$

where $\Phi_{0}$ is the superconducting flux quantum. We have found $\xi_{\|}(T=0)=7.1 \mathrm{~nm}$ for $(1 \mathrm{~nm} \mathrm{Si} / 1 \mathrm{~nm} \mathrm{~W}) \times 10$ and $\xi_{\|}(T=0)=9.1 \mathrm{~nm}$ for $(5 \mathrm{~nm} \mathrm{Si} / 1 \mathrm{~nm} \mathrm{~W}) \times 9$ MLs.

From the parallel critical fields the dimensionality and strength of coupling between the superconducting layers can be evaluated. For an anisotropic 3D superconductor

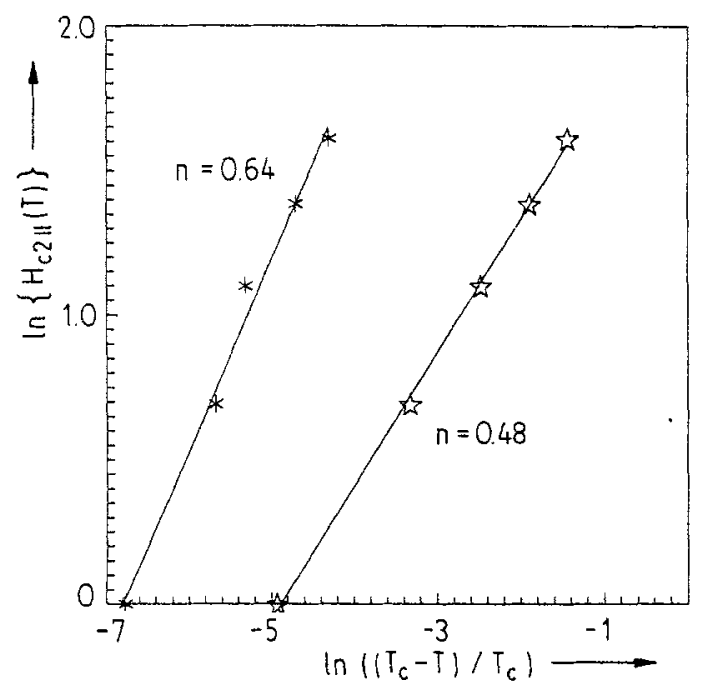

Fig. 6. Parallel critical field exhibiting a $\left(T_{c}-T\right)^{n}$ dependence with $n=0.48$ for $(1 \mathrm{~nm}$ $\mathrm{Si} / 1 \mathrm{~nm} \mathrm{~W}) \times 10$ and $n=0.64$ for $(5 \mathrm{~nm} \mathrm{Si}$ ' $1 \mathrm{~nm} \mathrm{~W}) \times 9$ multilayers 
one can write [1]

$$
H_{\mathrm{c} 2 \|}(T)=\frac{\Phi_{0}}{2 \pi} \frac{1}{\bar{\zeta}_{\|}(T) \zeta_{\perp}(T)}
$$

with coherence lengths $\xi_{\perp}(T), \xi_{\|}(T) \sim\left(T-T_{c}\right)^{-1 / 2}$.

For a 2D superconductor [22],

$$
H_{\mathrm{c} 2 \|}(T)=\frac{\Phi_{0}}{2 \pi} \frac{\sqrt{12}}{t} \frac{1}{\xi_{\|}(T)} .
$$

Here $t$ is the thickness of the superconducting layer. The experimental data of $H_{\mathrm{c} 2 \|}(T)$ revealed the $H_{\mathrm{c} 2 \|}(T) \sim\left[\left(T_{\mathrm{c}}-T\right) / T_{\mathrm{c}}\right]^{n}$ dependence (Fig. 6) with the values of $n=0.48$ for $\cdot(1 \mathrm{~nm} \mathrm{Si} / 1 \mathrm{~nm} \mathrm{~W}) \times 10$ and $n=0.64$ for $(5 \mathrm{~mm} \mathrm{Si} / 1 \mathrm{~nm} \mathrm{~W}) \times 9$. These values are close to 0.5 which is typical for $2 \mathrm{D}$ superconductors. Considering that the condition $(t \ll \xi)$ is fulfilled for both samples, the coupling strength can be evaluated by the calculation of the effective layer thickness $t$ using (4). This value correlates with the perpendicular coherence length. For the sample $(1 \mathrm{~nm} \mathrm{Si} / 1 \mathrm{~nm} \mathrm{~W}) \times 10 t=15.2 \mathrm{~nm}$, which is close to the total multilayer thickness (Table 1). For the sample $(5 \mathrm{~nm} \mathrm{Si} / 1 \mathrm{~nm} \mathrm{~W}) \times 9 t=2.89 \mathrm{~nm}$ is obtained. This value is lower than the multilayer period (Table 1) in agreement with the assumption of thin alloyed $\mathrm{W}-\mathrm{Si}$ superconducting layers and indicates very weak coupling between the superconducting layers in this sample.

Summarizing the critical field data, both multilayers behave as $2 \mathrm{D}$ type-II superconductors. The $(5 \mathrm{~nm} \mathrm{Si} / 1 \mathrm{~nm} \mathrm{~W}) \times 9 \mathrm{ML}$ behaves as a layered superconductor consisting of $2 \mathrm{D}$ superconducting layers with very weak coupling between them. The $(1 \mathrm{~nm} \mathrm{Si} /$ $1 \mathrm{~nm} \mathrm{~W}) \times 10 \mathrm{ML}$ reveals a strong interaction between the superconducting layers, however, due to the low number of periods $(N=10) 2 \mathrm{D}$ behaviour of the total ML stack is still observed.

In Fig. $3 \mathrm{~b}$ and $4 \mathrm{~b}$ a significant broadening of the resistive transition of $(1 \mathrm{~nm} \mathrm{Si} /$ $1 \mathrm{~nm} \mathrm{~W}) \times 10$ and $(5 \mathrm{~nm} \mathrm{Si} / 1 \mathrm{~nm} \mathrm{~W}) \times 9 \mathrm{MLs}$ in a magnetic field perpendicular to the superconducting layers is observed. When the magnetic field is applied parallel to the superconducting layers only negligible broadening of the resistive transition is found. As is manifested in $H_{\mathrm{c} 2\|\|}$ versus $T$ dependences for both samples, the effect of a parallel magnetic field in superconducting transition is more pronounced for the strongly coupled sample $(1 \mathrm{~nm} \mathrm{Si} / 1 \mathrm{~nm} \mathrm{~W}) \times 10$.

The broadening of the resistive transition in perpendicular magnetic fields is intensively studied at present for highly anisotropic superconducting systems like high- $T_{\mathrm{c}}$ superconductors, superlattices of high- $T_{\mathrm{c}}$ superconductor/semiconductor type, and multilayers of superconductor/nonsuperconductor type. The data are discussed mainly in the framework of the flux creep model given by Anderson [23] and Kim et al. [24]. The temperature dependences of the resistivity $\varrho(T, H)$ below $T_{\mathrm{c}}$ in external magnetic fields are analyzed using the Arrhenius relation

$$
\varrho(T, H)=\varrho_{0} \exp \left\{-\frac{U(T, H)}{k_{\mathrm{B}} T}\right\},
$$

where $\varrho_{0}$ is a constant, $U(T, H)$ is the activation energy for magnetic flux line motion.

Palstra et al. [7] analyzed the thermally activated behaviour of $\mathrm{YBaCuO}, \mathrm{BiSrCaCuO}$, and $\mathrm{TlBaCaCuO}$ high $-T_{\mathrm{c}}$ superconductors with different degrees of anisotropy. The authors 
found a close relation between the value of the activation energy $U$ and the degree of anisotropy of the material. The largest $U \approx k_{\mathrm{B}}\left(10^{4}\right.$ to $\left.2 \times 10^{5}\right) \mathrm{K}$ for magnetic fields up to $12 \mathrm{~T}$ was reported for $\mathrm{YBaCuO}$. Here, also the temperature dependence of the activation energy has to be taken into account. Much smaller activation energies $U \approx k_{\mathrm{B}}(300$ to 3000$) \mathrm{K}$ for magnetic fields up to $12 \mathrm{~T}$ were found for $\mathrm{BiSrCaCuO}$ and $\mathrm{TlBaCaCuO}$ superconductors with higher degree of anisotropy. From the Arrhenius plot of $\ln R$ versus $1 / T$ a constant value of $U$ over a limited temperature interval was inferred. For these superconductors a magnetic field dependence of the activation energy $U \sim H^{-\alpha}$ with $\alpha=0.25$ to 0.3 was observed.

Brunner et al. [6] analyzed the thermally activated flux motion in $\mathrm{YBaCuO} / \mathrm{PrBaCuO}$ superlattices with different thicknesses of the $\mathrm{PrBaCuO}$ layers thus varying the coupling strength between the superconducting layers. The authors found the same activation energy for superlattices with different insulating layers thick enough to suppress the interlayer coupling. Larger activation energies were found for superlattices with thinner insulating layers when the interlayer coupling was introduced. The dependence of the activation energy $U$ on magnetic field was of $U \sim-\ln H$ type. A similar increase of $U$ with decreasing $\mathrm{PrBaCuO}$ layer thickness was reported also by Jakob et al. [5] for $\mathrm{YBaCuO} / \mathrm{PrBaCuO}$ superlattices. Here, the temperature independent activation energy depends on the $\mathrm{PrBaCuO}$ layer thickness $t_{1}$ as $U \sim t_{1}^{-6}$. The magnetic field dependence of $U$ can be expressed as $U \sim H^{-x}$ with $x=0.5$.

The broadening of the resistive transition in magnetic fields was observed also for low-temperature superconductors. Graybeal and Beasley [25] observed the broadening of the resistive transition in magnetic fields for $2 \mathrm{D}$ superconducting amorphous MoGe thin films. They analyzed the $\varrho(T, H)$ data below $T_{c}$ as thermally activated process with constant activation energy $U(H)$ when the magnetic field is fixed. The field dependence $U \sim H^{-2 / 3}$

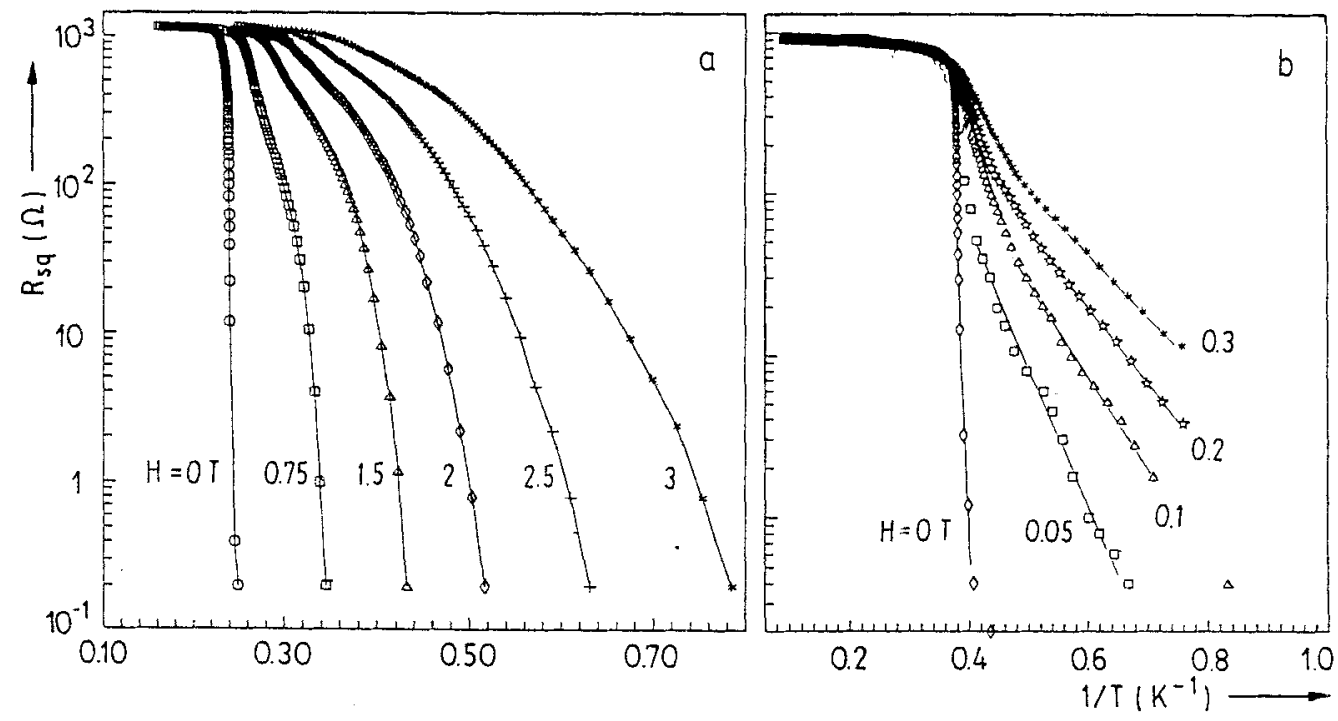

Fig. 7. Arrhenius plot of the sheet resistivity for selected magnetic fields for a) $(1 \mathrm{~nm} \mathrm{Si} / 1 \mathrm{~nm} \mathrm{~W}) \times 10$ and b) $(5 \mathrm{~nm} \mathrm{Si} / \mathrm{nm} \mathrm{W}) \times 9$ multilayers 


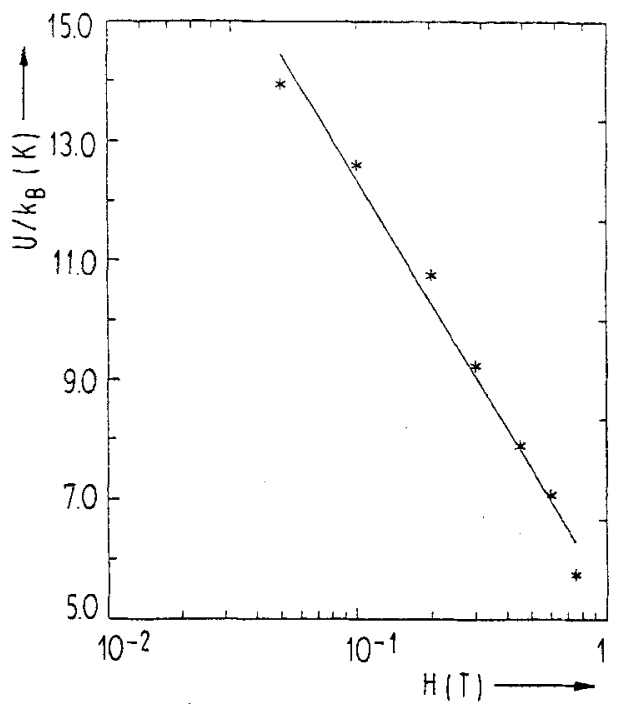

Fig. 8. Magnetic field dependence of the activation energy $U$ for the $(5 \mathrm{~nm} \mathrm{Si} / 1 \mathrm{~nm} \mathrm{~W}) \times 9$ sample

was found. The authors described this behaviour which is different from flux flow or depinning phenomena as a universal behaviour of 2D superconductors. Garland and Lee [26] observed thermally activated behaviour below $T_{c}$ for $\mathrm{In} / \mathrm{InO}_{x}$ composites. For a fixed magnetic field a slowly temperature dependent activation energy $U$ was found. The dependence $U \sim 1 / H$ was obtained up to a magnetic field $0.1 \mathrm{mT}$, for higher fields $U$ was found to be independent of $H$. Recently, the broadening of the resistive transition was reported for MoGe/Ge MLs by White et al. [8]. The MoGe/Ge MLs with different $\mathrm{Ge}$ layer thicknesses represent a coupled 2D superconducting system with different coupling strength similar to the present $\mathrm{W} / \mathrm{Si}$ system. Below $T_{\mathrm{c}}$ thermally activated behaviour affected by the interlayer coupling strength was found. For more strongly coupled MoGe/Ge MLs the authors observed very similar behaviour as was reported for the $\mathrm{YBaCuO}$ high- $T_{\mathrm{c}}$ superconductor by Palstra et al. [7]. For less strongly coupled multilayers the activation energy $U(0.92 \mathrm{~T})=k_{\mathrm{B}} \cdot 195 \mathrm{~K}$ was calculated from the Arrhenius plot.

Fig. $7 \mathrm{a}$ and b show the Arrhenius plots of the resistive transitions of $(1 \mathrm{~nm} \mathrm{Si} / 1 \mathrm{~nm} \mathrm{~W}) \times 10$ and $(5 \mathrm{~nm} \mathrm{Si} / 1 \mathrm{~nm}$ W) $\times 9$ multilayers in perpendicular magnetic fields. Different behaviour can be observed for W/Si MLs with different interlayer coupling strength. For the weakly coupled $(5 \mathrm{~nm} \mathrm{Si} / 1 \mathrm{~nm} \mathrm{~W}) \times 9$ ML the activation energy $U$ of flux motion was calculated (6) from the straight lines of the Arrhenius plots. The dependence of the activation energy on magnetic field is roughly $U \sim-\ln H$ between 0.1 and $1 \mathrm{~T}$ as shown in Fig. 8. For the more strongly coupled multilayer $(1 \mathrm{~nm} \mathrm{Si} / 1 \mathrm{~nm} \mathrm{~W}) \times 10$ the $\ln R$ versus $1 / T$ data exhibit a strong curvature and it is not possible to extract an activation energy independent of temperature. This behaviour is similar to that mentioned above for anisotropic superconductors and superconducting superlattices and multilayers. A temperature independent activation energy is mostly observed for weakly coupled layered superconducting systems. Generally, for more strongly coupled superconductors the dependence of the superconducting parameters on the temperature is more pronounced.

As mentioned above, two different types of dependences of the activation energy $U$ on the magnetic field were reported up to now, i.e. $U \sim-\ln H$ or $U \sim H^{-\alpha}$. The origin of this difference is still a subject for discussion. Our results show the $U \sim-\ln H$ dependence. We suppose that the effect of vortex fluctuations on the in-plane resistivity of $2 \mathrm{D}$ superconductors recently published by Jensen et al. [27], could explain our results. The authors have shown that the thermally activated behaviour with the activation energy $U \sim-\ln H$ could be a consequence of unbinding vortex pairs at temperatures close to $T_{\mathrm{c}}$. 


\section{Conclusion}

The effect of different coupling strengths between the superconducting layers on superconducting parameters was studied for amorphous $\mathrm{W} / \mathrm{Si}$ multilayers. We observed a nearly linear increase of $T_{\mathrm{c}}$ with increasing number of bilayers up to $N=10$. An increasing anisotropy of the critical fields $H_{\mathrm{c} 2 \downarrow}, H_{\mathrm{c} 2} \mid$ with increasing Si layer thickness was found. The data suggest that the $(5 \mathrm{~nm} \mathrm{Si} / 1 \mathrm{~nm} \mathrm{~W}) \times 9$ multilayer is a $2 \mathrm{D}$ layered superconductor with very weak coupling between the superconducting layers. The $(1 \mathrm{~nm} \mathrm{Si} / 1 \mathrm{~nm} \mathrm{~W}) \times 10 \mathrm{ML}$ is a $2 \mathrm{D}$ strongly coupled layered superconductor. Here, the $2 \mathrm{D}$ behaviour is due to the low total multilayer thickness. Resistive broadening in perpendicular magnetic fields was observed for both weakly and strongly coupled multilayers with simple Arrhenius-type flux flow with temperature independent activation energy observed for the weakly coupled ML only.

\section{Acknowledgements}

This work was supported by Slovak Grant Agency and in part by the Deutsche Forschungsgemeinschaft. One of authors (S. L.) acknowledges the support from the Alexander von Humboldt Foundation.

\section{References}

[1] S. T. Ruggiero and M. R. Beasley, in: Synthetic Modulated Structures, Ed. L. L. Chang and B. C. GIESSEN, Academic Press, 1985 (p. 365).

[2] J.-P. Locquet, D. Neerinck, H. Vanderstraten, W. Sevenhans, C. Van Haesendonck, Y. BRUYNSERAEDE, H. Homma, and I. K. SChuller, Japan. J. appl. Phys. 26, 1431 (1987).

[3] D. Neerinck, K. Temst, C. Van Haesendonck, Y. Bruynseraede, A. Gilabert, and I. K. SCHULLER, Europhys. Letters 15, 637 (1991).

[4] Q. LI, X. X. XI, X. D. Wu, A. Inam, S. Vadlamannate, W. L. Mclean, T. Ventakesan, R. Ramesh, D. H. Hwang, J. A. Martinez, and L. Nazar, Phys. Rev. Letters 64, 3086 (1990).

[5] G. JaKob, T. HahN, C. Stolzel, C. Tome-Rosa, and H. Adrian, Europhys. Letters 19, 135 (1992).

[6] O. Brunner, L. Antognazza, J. M. Triscone, L. Mieville, and O. Fischer, Phys. Rev. Letters 67, 1354 (1991).

[7] T. T. M. Palstra, B. Batlogg, R. B. van Dover, L. F. Schneemeyer, and J. V. Waszczak, Phys. Rev. B 41, 6621 (1990).

[8] W. R. White, A. Kapitulnik, and M. R. Beasley, Phys. Rev. Letters 66, 2826 (1991).

[9] S. Luby, E. Majkova, P. Lobotka, I. Vavra, M. Jergel, R. Senderak, and J. Grno, Physica (Utrecht) $197 \mathrm{C}, 35$ (1992).

[10] C. KitTel, Introduction to Solid State Physics, Wiley, New York 1971 (p. 402).

[11] K. MaEX, Mater. Sci. Engng. R11, 53 (1993).

[12] E. Majkova, P. Lobotka, I. Vavra, S. Luby, M. Jergel, S. Benacka, R. Senderak, B. George, and M. Vaezzadeh, Appl. Surface Sci. 65/66, 752 (1993).

[13] M. Jergel, E. Majkova, and S. Luby, J. Physique IV, Coll. C83, 337 (1993).

[14] M. Brunel, S. Enzo, M. Jergel, S. Luby, E. Majkova, and I. Vavra, J. Mater. Res. 8, 2600 (1993).

[15] M. Strongin, O. F. Hammerer, D. H. Douglas, and M. H. Cohen, Phys. Rev. Letters 19, 3086 (1990).

[16] R. F. Frindt, Phys. Rev. Letters 28, 299 (1972).

[17] J. M. Triscone, O. Fischer, O. Brunner, L. Antognazza, A. D. Kent, and M. G. Karkut, Phys. Rev. Letters 64, 804 (1990).

[18] A. Odagawa, K. Setsune, T. Matsushima, and T. Fujita, Phys. Rev. B 48, 12985 (1993). 
[19] K. Michielsen, T. Schneider, and H. DeRaedt, Z. Phys. B 85, 15 (1991).

[20] E. Majkova, S. Luby, M. Jergel, R. Senderak, and B. George, Thin Solid Films 238, 235 (1994).

[21] J. Z. Wu, X. X. Yao, C. S. Ting, and W. K. Chu, Phys. Rev. B 46, 14059 (1992).

[22] M. Tinkham, Phys. Rev. 129, 2413 (1963).

[23] P. W. ANDERSON, Phys. Rev. Letters 9, 309 (1962).

[24] Y. B. Kim, C. F. Hemptstaed, and A. R. Strnad, Phys. Rev. 131, 2486 (1963).

[25] J. M. Graybeal and M. R. Beasley, Phys. Rev. Letters 56, 173 (1986).

[26] J. C. Garland and Hu Jong Lee, Phys. Rev. B 36, 3638 (1987).

[27] H. J. Jensen, P. Minnhagen, E. Sonin, and H. Weber, Europhys. Letters 20, 463 (1992).

(Received June 2, 1994) 